\title{
How to Instill Clinical Trials Culture in Participants of Developing Countries
}

\author{
Ndje Ndje Mireille \\ Faculty of Arts Letters and Human Sciences PO.Box 755 Yaoundé, Cameroon
}

\begin{abstract}
This work aims at finding clues that can stimulate the availability and motivation of the populations of developing countries to participate in a voluntary and spontaneous manner in biomedical research. A research team and participants were evaluated via a project monitoring visit on the means used to conduct a clinical trial in which the Kumba District Hospital was one of the sites. The research team has been trained in Good Clinical Practices, and has well explored the Protocol with the promoters. The team was trained in the administration of the free and informed consent. It has conducted sensitizations campaigns in the hospital, and also in communities. Participants were well informed on the trial, the risks and their freedom to participate and to interrupt their consent at any time without risk of reprisal. This has facilitated access of participants to this clinical trial, however, no spontaneous participation was recorded. Mastery of the protocol by the research team and a good sensitization of the population might be drivers of a culture of voluntary and spontaneous participation in clinical trials in developing countries.
\end{abstract}

Key words: Clinical trial, research, voluntary participation, sensitization, participants.

Abbreviations: AEM, Agence Européenne des Médicaments; CNEC, Comité National d’Ethique du Cameroun; EDCTP, European and Developing Countries Clinical Trials Partnership; FDA, Federal Drug Administration; KDH, Kumba District Hospital; LSHTM, London School of Hygiene and Tropical Medecine; OMS, Organisation Mondiale de la Santé; WHO, World Heath Administration; P.I., Principal Investigator; HIV, Human Immunodeficiency Virus.

\section{Introduction}

Epidemics are a recurring phenomenon in Africa. HIV/AIDS, malaria, tuberculosis, Ebola virus, and many others constitute a real threat to african countries that desperately need to grow. These epidemics make Africa a fertile field for biomedical research. The youth of ethics committees in Africa and sometimes their virtual absence from some countries encourage the violation of rights and human dignity of participants in different research projects. Since the introduction of a reflection on ethical issues $\left.{ }^{[1}\right]$, in the world of health and biomedical research back in the 1960s the abuse on human beings during research are denounced. However, some research projects, particularly clinical trials were conducted in Africa ignoring international ethical standards. A

Corresponding author: Ndje Ndje Mireille, clinical psychologist, research fields: clinical psychology, perinatal clinic, research ethic, social sciences methodology. clinical trial is a biomedical research organized and practiced on human beings to develop biological or medical knowledge. Some clinical trials focus on drugs and seek to establish or verify pharmacokinetic data (terms of absorption, distribution, metabolism and excretion of the drug), pharmacodynamic (mechanism of action of the drug including) and therapeutic (efficacy and tolerance) of a new drug or a new way to use a treatment that exists. There are some researches that have been conducted in Africa. The medical research on microbicides, with a trial on the cellulose sulphate, was capped by CONRAD in Benin, India, South Africa and Uganda [2]. According to the [2], this test was interrupted before completion due to the higher number of HIV infections that was found in the group using the product compared to the placebo group. In Cameroon, one Randomized placebo trial that sought to test the ability of the drug Tenofovir to prevent contamination of HIV infection was 
conducted on 400 prostitutes [3]. Half of the participants received placebo, but felt protected and went out without their usual methods of protection. Each participant received the puny sum of four euro to engage in the trial. Tsala Tsala (2008) [3] shows that in any test of prevention, protection induced feeling of risk behaviors. We can also note a total disinterest of some participants in clinical trials. They are usually attracted by the low amount of money that is offered to them, or even the free treatments that they are proposed. They seem no more interested in the study in which they participate, on the scope that the study can have on them and even the whole community, still less to the ethical issues of the projects. Monitoring visits conducted by the Cameroon National Ethics Committee in 2013 during the CNEC-EDCTP No. CB.10.41302.008 project "Strengthening the Cameroon National Ethics Review Committee” has identified the difficulty of researchers to carry out clinical trials in the field. The vast majority of participants at the various sites were not even aware that they were participating in a research. They did not make the difference between their regular medical care and research, they did not know what a clinical trial was, they often mistook it for a medical treatment, and however, they were aware of their rights or duties. Some participants highly feared side effects of the drugs they were going to receive. To these are added the barriers and prejudices lurking on Biomedical Research (desire to make people sterile, inoculation of viruses, exploitation of black people, researchers desire to enrich themselves at the expense of the poor ...) talkless of the impact of culture that often does not allow women and children to engage in research. Shah (2007) [4] argues that for clinical trials, essential before the sale of new drugs, pharmaceutical companies are turning to the South. They are many and more docile patients, as well as ethical and legal constraints often are less stringent than in rich countries. If instead of alternative, the participants in these trials can take advantages, this new form of exploitation of the body leads to a disturbing relocation of risks ... without much consideration. We could then try to question the reliability of clinical trials carried out in this context. Yet, as Thompson (2003) [5] says, it is normal that biomedical researches conducted in developing countries increased. According to Article 24 of the Helsinki Declaration on medical research on human beings, each potential participant to be involved in a clinical trial should be properly informed about the objectives, methods, sources of funding, any possible conflict of interest, institutional affiliations of the researcher, the anticipated benefits and potential risks of the study and the discomfort it may entail, and any other relevant aspects of the study. The need for participants in clinical trials to have as much information on research projects, to learn about the protocols, to be informed about the risks and benefits of trials in which they participate proves so vital. Better still it would be interesting for researchers to instill a culture of clinical trials to African (african) people called to participate in these clinical trials. The EDCTP-CNEC project allowed CNEC to conduct monitoring visits to sites where projects were home at the CNEC to evaluation. Of these visits, it appears that, on 15 sites visited, one has managed to conduct the clinical trial on 'Tranexamic Acid for the Treatment of Postpartum Haemorrhage': An international randomized, double blind, placebo controlled trial, promoted by the LSHTM (London School of Hygiene and Tropical Medicine). The Principal investigator for the site was Doctor Etienne Asonganyi Defang Ngunyi, obstetrician gynecologist in the said hospital, assisted by several other co-investigators but especially nurses and midwives. Through the preparation that was made to potential participants, the trial went on easily. This is the KDH (Kumba District Hospital) in South West Cameroon, visited on May 24th 2013, which has served us as a model to suggest how to instill clinical trials culture in participants of developing countries. 


\section{Methods}

In order to emphasize on the factors that can help to instill a clinical trial culture in participants of developing countries, we went through an analysis of the founding of the monitoring of the project "Tranexamic Acid for the Treatment of Postpartum Haemorrhage": An international randomized, double blind, placebo controlled trial in Kumba District Hospital. A study is randomized when patients are divided into different groups receiving different treatments. This distribution between the groups is achieved by randomization also called draw. The monitoring conducted by the National Ethics Committee of Cameroon on May 24, 2013. The visit began with routine activities that were framed around the guided tour of the hospital, meeting with those involved in the project, the presentation of the monitors and the statement of the purpose of the visit, the visit of the premises of the site (offices, delivery room), consultation of documents and archives of the project (by the team of LSHTM), participation in a prenatal animation session. According to the requirements of the National Ethic Committee of Cameroon in monitoring, two sets of themes have been retained and will guide us to conduct this study. The themes are addressed to members of the project team and to the research participants.

For members of the research team we are interested in:

- Recruitment conditions

- Organization of the work

- Knowledge on the study protocol

- Characteristics of the trial product

- Ethical consideration

- Good Clinical Practice

For participants

- Knowledge of participants on the study (objectives, risks...)

- Conditions in which informed consent is obtained

- Perception of the study by participants
- Fair selection of participants for study in double blind

- Voluntariness of the participation

- Right to withdraw one's participation without any penalty

\section{Results}

For the activities carried out before the actual monitoring, it may be noted that the project is housed within the maternity of the hospital. The hospital authorities accepted to host the project and all the staff of the hospital was notified. The study was managed by the Principal investigator, obstetrician gynecologist who was assisted by other physicians but especially nurses and midwives. The folders are neatly in cabinets, products are well preserved and records are well maintained.

\subsection{Recruitment Conditions}

Beyond the criteria of the CNEC, the research team of the $\mathrm{KDH}$ took the initiative to educate pregnant women in advance through the distribution of leaflets, posters, information during prenatal visits, educational talks to patients who attended the hospital, even those who were not likely to participate in the study, sensitization in the communities. Representatives of patients received leaflets and other information about the study, and they had the opportunity to ask questions. All women who gave birth in the hospital during the study period were supposed to participate in the study. Participants were recruited after childbirth if they had postpartum hemorrhage and especially if they agreed to participate in the study. When they were aware, a nurse would administer the free and informed consent. The participant received first normal processing and was then included in the study.

\subsection{Organization of the Work}

The archives are kept, classified, and secured. Communication between researchers and potential 
participants during prenatal period is dynamic, even though we may note some biases in the communicated information, especially with regard to the notion of research. The documents we found in the site of the project are the same as those filed with the Ethics Committee (clearance, insurance...). Patients folders are neatly in the hospital. (Archives) are well preserved. All files of participants included in the study can be found. The normal medical was provided to participants the same way it was provided to hospital patients. The nurse came in the morning and began their normal working day. When a potential participant arrived, one of the nurses would take care of her. A rotational program was developed for members of the team. Recruitment was done every day and continued in the night during the guards for pregnant women who arrived in the night. The PI was responsible for coordinating the fieldwork and ensuring strict compliance with ethical standards.

\subsection{Knowledge on the Study Protocol}

The site of KDH has also put a focus on the training of the hospital staff. Even when he was not part of the research team, he could guide potential participants. A particular emphasis was placed on the training of team members (the appropriation of the Protocol, training in the Good Clinical Practice, respect of the procedure for protecting confidentiality, administration of informed consent). A nurse at the hospital said: "The project gave me a culture of documentation and allowed us to learn how to sensitize.” This solid training enabled the research team to explain to potential participants the ins and outs of the Protocol and the whole project. This impregnation has facilitated the participation of women meeting the criteria of inclusion at the $\mathrm{KDH}$ in this clinical trial. The members of the team were aware that the interests of participants will always prevail over those of science and society. Moreover, proponents have designed the research in such a way that minimizes pain, physical and psychological discomfort.

\subsection{Characteristics of the Trial Product}

The team members have demonstrated a good knowledge of the product that was administered to the participants. A nurse well described the particularity of the product that she administered to the participants in these terms: "'placebo may be administered to a participant without that she knows what kind of treatment she takes. It is called blind trial. Myself I can ignore it and in this case this is the double-blind trial'. Indeed, in this type of trial, there are two groups of patients. One receives the new treatment to be evaluated and the other receives a placebo, a substance having the same format as the drug but that is inactive. This comparison of the two groups helps to measure the effectiveness of the new treatment. People who received placebo do not have any personal benefit from the research. The product can just benefit to patients under treatment. However the participation of the placebo group is necessary to validate the new treatment.

\subsection{The Ethical Consideration}

The promoter of this clinical trial has provided coverage for any damage that may arise in connection with the research. The confidentiality of the personal data in connection with the research is also guaranteed. If the results were to be published, it would be on an anonymous basis. The participant is informed that the competent health authorities, as well as representatives of the sponsor and the CNEC members will have access to his personal data to check the proper conduct of the research. They knew that these data are not held secret. The research is carried out in an appropriate framework and by competent persons. The selection of subjects is equitable. The research has received ethics clearance of CNEC whose members, experts from clinical trials, representatives of other disciplines such as bioethics, public health, pharmacology, human and social science has carefully examined the Protocol. The Committee has evaluated the balance risk-benefit for participants in this 
research. The sponsor paid for insurance guaranteeing civil liability, in order to allow, in case of damage due to research, compensation to entrant. He also attested to be responsible for the proper conduct of the research and the data quality, hence its many field visits to evaluate the progress of the research

\subsection{GCP (Good Clinical Practice)}

The research team nurses all followed training in GCP online. Consent sheets, incomes and the outcomes forms were well filled and updated. The stock of medicines and the archives were well preserved. The project has recorded no refusal of participation

\subsection{For Participants}

3.7.1 Knowledge of Participating on the Study (Objective, Risks...)

We found three participants on the site during our visit. We were not able to meet a representative of the participants who could provide us with information on its own knowledge of the draft, given that some participants were recruited in a State of unconsciousness and therefore the first consent has been given by a representative. It emerged that participants and their representatives are knowledgeable about the research, the procedures, on the modalities of consent, and on their freedom to participate or not to the trial. One participant stated being well informed on research and its procedures, on free and informed consent and he clearly affirmed "I knew that I am free to accept or refuse to participate in the research". Participants knew they were participating in a very different care from routine medical care. They also knew that it was to test the effectiveness of a new product and that it was a double-blind trial. This implies that some participants were receiving just placebo. They were also reassured that they received in advance their normal treatment.

3.7.2 Conditions in which Informed Consent Is Obtained

Following the German and Japanese medical research during the Second World War and on the occasion of the Nuremberg trials, one of the first internationally recognized regulations was established. This is the Nuremberg Code (1947) [6]. This Code often rules, determines the conditions necessary for a research on humans to be acceptable, and the free and informed consent of the participant here is the key standard and becomes the prerequisite for any research involving human beings. Freedom of participants to participate in this research is quite clear in this trial on the site of the $\mathrm{KDH}$. They confirm by written consent after receiving the complete, oral and written information on the nature, the benefits and the risks associated with the study in question. In this type of clinical trial, that is randomized double-blind, the researcher and the patient do not know what treatment is given. The approach has been explained to each of the participants, and even other women attending the hospital. The participants were also informed that, beyond the trial product, they received the normal treatment of post partum hemorrhage. During the trial, some participants were not able to give free and informed consent because of their State of health. In this situation a legal representative of the participant consented. In some cases of extreme emergency, relatives were consulted after the fact in order to establish what would have been the will of the participant. The research team has vigil that the information on the consent form be clear and understandable to the patient. The team members have sometimes expressed themselves in local languages to promote exchange and assist participants in decision making. Consent was evidenced by the signing of a consent form indicating that the person has received all the information needed about the clinical trial and that he agreed with all that the information on the consent form.

3.7.3 Perception of the Study by Each Participant

For this participant met at the hospital, the research finally has other aims than the profit of the researcher "before, I thought that white people who come to 
research in Africa have a lot of money, we just support. It is after when the nurse explained that I understood that it was to help us too and that if the test works well, we'll save more women who give birth and bleeding." The majority of participants were septic about the research, because of prejudices lurking in their environment. Also, many were unaware of even the notion of research and said that they had never imagined that it was to test a drug before its release. Some have suggested that they had believed that they will earn a lot of money in the project, that's why they have decided to listen. It should be noted that despite these blocks initially faced while searching, the flow of information received at the preparatory phase of the trial has helped people in Kumba to have better readability in biomedical research. Many participants have promised to give their consent whenever they will be solicited to participate in research.

\subsection{Voluntariness of the Participation}

Clinical trials play an essential role in the development of new treatments, the inclusion of volunteers is essential. Low recruitment in clinical trials slows progress and discourages potential investment. Several clinical trials are delayed because of the difficulty in finding volunteers and many others do not settle. In the same project, we noticed garish failures on other sites. Clinical trials protocols usually involve people responding to their selection criteria. Persons who do not meet the selection criteria are not included for their safety, but for the good conduct of the trial and the reliability of the results also. Participation in a clinical trial is voluntary. The participant must be informed and must consent. In this trial, objectives, duration and methodology of the trial were well known by the participants. In other contexts, the volunteers are recruited through ads in newspapers, on the Internet, in universities (including medical faculties), in hospitals. Participating in a clinical trial requires a minimum of availability and motivation.

\subsection{Right to Withdraw One's Participation without Any Penalty}

The team members know that the protection of participants in a clinical trial is guaranteed by law, while in Cameroon in particular, there is still no law on the matter. A nurse said "we are required to inform the participants of their state of health during and at the end of the trial. When the test is complete, they must be informed of the results of research.” The nurses told us that participants have the right to suspend their participation in the trial at any time, without any liability or harm. It should be noted that at any time the research participant may withdraw from the trial, without giving a reason but without incurring damage. Its management must continue without consequences. Participants knew they could check the data on and could [7] oppose their transmission. They had, for those who were aware, enough time to think before deciding to participate in the study.

\section{Conclusion}

Sensitizing populations even when there is no current project could prepare them for participation in clinical trials in a context where the support of the population remains problematic. At the end of this research, it was clearly established that any person may be asked to participate in a clinical trial, either during a visit to the doctor, by posting, by advertisement or through the media. People could also participate voluntarily and independently and contact research structures if they are sensitized regularly. We didn't meet any volunteer highlighting their curiosity and desire to help advance research. It suggests that Cameroonian participants still need motivation. Some highlights of this study at the KDH can be considered as the drivers of the culture of clinical trials in developing countries. It would be interesting to develop and disseminate a practical guide on the rights of participants in health research, especially those involving human beings for their own purpose, but also for family members, their entourage and even the 
general public. This guide would focus on informing them on issues of ethical order and address their concerns in this regard and especially to motivate them to participate in health research. The other benefits over financial and material must be highlighted (advances in research, new treatments, health promotion). The participants' consent should always be obtained if possible before administering the product. The team should clearly explain to participants that it is a research and they are not in the usual practice. Participants should be aware that this is a trial, not a treatment. The research team should receive more training and be provided with adequate working equipment (computers, internet ...). Members of the research team should be easily identifiable. After completing the test, the clinical research team should publish all findings and make them available. The AEM (Agence Européenne des Médicaments) and his US counterpart the FDA (Food Drug Administration) requested that the test results are published in a scientific or medical journal and made public in accordance with the amendment law of the
FDAA (Food Drug Administration) in 2007.

\section{References}

[1] Durand, V. M. 1999. General Introduction to Bioethics. History, Concepts and Tools. Montréal et Paris, Fides et Cerf, 572. (in French)

[2] Aline, R. 2008. What Has Changed in Science. http://www.larecherche.fr.

[3] Tsala Tsala, J. P. 2008. "When Researchers Come from the North.” Tenofovir Trials Cases in Cameroon. III Meeting of Francophone International Bioethics. Quebec. (in French)

[4] Shah, S. A. 2007. Ethnomedical Best Practices for International Psychosocial Efforts in Disaster and

[5] Trauma. In E. Tang and J. Wilson (Eds.) Cross-Cultural Assessment of Psychological Trauma and PTSD, 51-64.

[6] Thompson, C. 2003. "Clinical Experience as Evidence in Evidence-Based Practice.” Journal of Advanced Nursing 43 (3): 230-7.

[7] Nuremberg Code 1947. Translations and Adaptations in French, in Guinea Pigs and Men: Experimentation on the Human Being and Justice, Paris, Belles Lettres, 2011. appendice électronique. http://descobayesetdeshommes.fr/Docs/NurembergTrad. (in French)

[8] CCNE Avis n 109. 04/02/2010. Communication of Scientific and Medical Information, and Society: Ethical Issues. http://www.ethique.inserm.fr /. 